\title{
Retos contemporáneos en la labor docente: ¿cómo es la situación en Latinoamérica?
}

\author{
Contemporary challenges in teaching: \\ What is the situation like in Latin America?
}

\author{
Iván Leonardo Medina Alvarado \\ Magíster en Administración de Empresas \\ Universidad Santo Tomás \\ Bogotá, Colombia \\ Correo electrónico: ivanmedina@ustadistancia.edu.co
}

Recibido: 1 de agosto de 2018

Aceptado: 11 de septiembre de 2018

Cómo citar este artículo:

Medina, A. (2019). Retos contemporáneos en la labor docente, ¿cómo es la situación en Latinoamérica? Espiral, Revista de Docencia e Investigación, 9(1), 183 - 194.

\section{Resumen}

La situación que viven los sistemas educativos en Latinoamérica y el mundo entero es el reflejo de los resultados obtenidos desde la gestión de las políticas educativas y la contextualización de la educación en relación con el desarrollo de las regiones. Es por lo que a continuación se describen algunos aspectos que tienen como intención identificar la labor de los docentes en este conjunto de cambios, que hacen de la educación un pilar fundamental de la sociedad contemporánea.

Para comprender las dinámicas actuales por las que la labor del profesorado está atravesando, este documento presenta tres retos para los docentes: a. La participación docente en las políticas educativas, para lo cual se analizan las situaciones que algunos países latinoamericanos comparten como sus avances en términos de vinculación docente en la agenda pública educativa. b. Formación académica en la labor docente, este reto se consolida como un punto clave en la cualificación docente y la objetividad de esta formación en la función transformadora de la educación. c. Nuevas competencias en el quehacer docente, quizás esta situación es un común denominador que con el transcurrir del siglo XXI se ha establecido como un reto para transformar la educación desde las aulas, teniendo en cuenta los contextos en los que el mundo globalizado está avanzando.

En última instancia, se comparten las conclusiones a las cuales se llega posterior a este análisis, junto con los referentes bibliográficos que confeccionan este escrito.

Palabras clave: Participación docente, formación docente, competencias docentes, políticas educativas, educación en Latinoamérica, profesorado.

\section{Abstract}

The situation experienced by education systems in Latin America and the entire world, the reflection of the results obtained from the management of educational policies and the contextualization of education for the development of the regions, is for this reason, which is described below some aspects that are intended to identify the work of teachers in this set of changes that make education a fundamental pillar of contemporary society.

To understand the current dynamics by which the work of teachers is going through, this document presents three important challenges for teachers, which are: a) Teacher participation in educational policies, for which the situations for which some are analyzed Latin American countries their progress in terms of teacher ties in the public educational agenda, b) Academic training in teaching work, this challenge is consolidated as a key point in teacher qualification and the objectivity of this training in the transforming function of education , and c) New competences in the teaching task, perhaps this situation is a common denominator that, with the passing of the 21st century, has been established as a replica to transform education from the classroom, taking into account the contexts in which the world Globalized is moving forward.

Ultimately, the conclusions reached after these analyses are included, together with the bibliographical references that make this writing.

Keywords: Teacher participation, teacher training, teacher competencies, educational policies, education in Latin America, teachers. 


\section{Introducción}

Las dinámicas del mundo actual están denotando cambios de todo tipo, es por esto que para Gisbert, Esteve-González, y Lázaro (2019) la educación no es la excepción, por tal razón, a continuación se presenta un análisis que se construye a partir de los retos que la labor del docente está enfrentando, desde los cambios que la sociedad está experimentando en el siglo XXI y como esta situación está afectando tanto positiva como negativamente el quehacer del docente en el aula y todo lo que implica la labor del profesorado desde el contexto latinoamericano.

Si bien es cierto que la labor docente en el interior de un centro educativo $y$, en general de un sistema educativo, concentra gran parte de la atención para con la dinámica administrativa, pedagógica y curricular. Marcelo (2010) comparte que de igual forma lo es el contexto bajo el cual se desarrollan las interacciones de los actores de una comunidad educativa, resultando ser puntos por considerar que van más allá de las fronteras del aula, y que convergen en comprender, entre otras cosas, la objetividad de las políticas públicas educativas, cuya administración y gestión recae en toda la comunidad, salvo que el contexto latinoamericano, por desgracia, denota muy poca participación de los docentes y los agentes educativos que hacen de la educación un punto clave de transformación, siendo precarios los escenarios de participación para construir conjuntamente planes, programas y proyectos que impacten una comunidad educativa, adicional, la formación académica en los docentes denota dos perspectivas, entre las cuales se encuentra, en primera instancia, formación académica descontextualizada con el quehacer del docente en el aula, y una segunda instancia posiblemente más compleja, en la cual no existe formación académica superior, dejando claro que ambos contextos son de libre albedrío en el docente, quien en última instancia es quien decide o no formarse académicamente y mejorar las competencias que su labor amerita.
Ahora bien, desde esta intención por comprender cómo se desarrolla la labor docente en el actual mundo globalizado, se busca comprender cuáles son las nuevas competencias que el docente debe manejar o hacer uso, ejemplo de esto, genera los siguientes cuestionamientos: ¿cómo se está usando por parte de los docentes las tecnologías de información y comunicación TIC?, ¿cómo se están fortaleciendo las habilidades investigativas en los docentes?, ¿en qué medida se están desarrollando las nuevas relaciones humanas desde el aula?, y ¿cómo la educación con pertinencia está incluyendo las nuevas herramientas que no solo están presentes en el contexto educativo, sino que están en la cotidianidad de los negocios, las comunicaciones, el cuidado por el medio ambiente y la construcción de las nuevas ciudadanías?

\section{Marco teórico}

En el escenario mundial, los cambios día a día se van consolidando como la situación clave para la innovación y el fomento de la creatividad, llegando a ser un punto de partida para intervenir y ajustar muchas de las tareas que la humanidad cotidianamente conoce, es así como Vaillant y Marcelo (2015) proponen que la educación también lo ha experimentado, lo está experimentando y con seguridad va a seguir experimentando este tipo de situaciones en las que el cambio es lo único seguro, y a partir del cual, la educación sin lugar a dudas seguirá siendo parte importante del desarrollo de los países y del mundo entero.

Desde esta perspectiva, la función que tienen todos y cada uno de los miembros de una comunidad educativa y que a la vez hacen parte de los sistemas educativos de cada país, resulta ser más que importante, toda vez que, un sistema educativo es el resultado de la interacción de múltiples escenarios y actores, quienes comparten una misma finalidad, la cual en el contexto educativo, se centra en la construcción colectiva del conocimiento, 
la trasformación de la sociedad a favor de la equidad y la igualdad $y$, antes que nada, favorecer el desarrollo integral del ser humano; de estos actores, El Plan Maestro (2015) comparte que el docente es un agente transformador que está directamente relacionado con todos los miembros de la comunidad educativa, siendo un canal de interacción entre directivos y estudiantes, administrativos y directivos, estudiantes y administrativos, y en general se configura como un engranaje en el interior de un centro educativo, resultando igual esta situación cuando se analiza desde la perspectiva de todo un sistema educativo.

Al ser claro que el docente es de suma importancia en todo un sistema educativo, este documento se construye para identificar tres situaciones o retos en las que de forma indispensable el docente debe ser reconocido como un protagonista, dejando claro que no son los únicos retos que se le presenta al docente desde su quehacer, pero que si procuran abarcar el impacto que la labor docente tiene en la educación, siendo estos tres retos los siguientes:

a) Participación docente en políticas educativas.

b) Formación académica en la labor docente.

c) Nuevas competencias en el quehacer docente.

\section{Participación docente en políticas educativas}

Una de las situaciones más complejas por las que un Gobierno debe atravesar es el alcanzar la participación de sus docentes en los procesos que no son necesariamente de enseñanza o en el aula, entendidos estos procesos como el conjunto de acciones que configuran y posibilitan la puesta en marcha de todo el sistema escolar y el funcionamiento mismo del aparato educativo; por lo tanto, tal como lo menciona Lozano y Mercado (2011), en el entorno latinoamericano es una realidad cada vez más cruda, en la que la resistencia por parte de muchos docentes es evidente por la poca o nula participación en escenarios colectivos en los que se procura que desde la perspectiva del docente se puedan intervenir algunas de las situaciones propias de las políticas educativas, como son:

- Optimización del sistema educativo.

- Mejoramiento y contextualización de la oferta educativa en las regiones.

- Carrera docente y labor docente.

Así las cosas, se presentan algunas de las siguientes propuestas para que el docente sea considerado como un participante activo de la agenda educativa, independientemente del nivel educativo en el actual esté la formación con la que cuente y la intención que tenga de participar, así las cosas se proponen las siguientes acciones:

\section{Conformación de mesas de trabajo}

Las mesas de trabajo se entienden como escenarios en los que se fijan temáticas por las cuales los docentes son organizados de acuerdo con una planeación previa, y tal como lo comparte Matute y Romo (2000), tienen como finalidad que los docentes desde entornos de comunicación colectiva, junto a compañeros y con la supervisión de algún tipo de supervisor o moderador discutan las situaciones complejas por las que está atravesando el sistema educativo; como resultado en estas dinámicas se espera que los docentes compartan sus experiencias generadas en el aula, desde el diario vivir y con una mirada más crítica de la realidad, que en muchas ocasiones se discute desde una junta directiva o una sesión, que desconoce concretamente las dinámicas que se construyen en el día a día del docente.

Para que las mesas de trabajo se consoliden como propuestas coherentes y efectivas para construir soluciones y no provocar situaciones adversas en las que los resultados tengan consecuencias nefastas, Medina, Pérez, y Campos 
(2014) proponen que estas mesas se deben acompañar de profesionales expertos que las dinamicen, cuya responsabilidad no se limite únicamente a acompañar cada sesión, sino que se prolongue para consolidar la información recolectada, para que esta sea organizada y presentada al director educativo que corresponda, como situación completaría. Estas mesas de trabajo no pueden ser generadas únicamente desde el escenario local, sino que deben ser estructuradas y planeadas desde tres perspectivas: a. mesas de trabajo locales, b. mesas de trabajo regionales, y c. mesas de trabajo nacionales; de manera que la información recolectada sea escalada a cada mesa de trabajo siguiente, y así las cosas, los gobiernos nacionales puedan tener de primera mano información vital para las intervenciones en los sistemas educativos y la actualización permanente de las políticas públicas en educación.

Lo interesante de este tipo de propuestas, es que la participación del docente está acompañada no solo por su director educativo, sino que esta contextualizada para las realidades que muchas veces son desconocidas en contextos como la ruralidad, la precariedad en infraestructura, la limitación de recursos, las condiciones culturales y las mismas dinámicas de las regiones y los ciudadanos; para Medina-Alvarado (2019) esta situación en Latinoamérica resulta ser una oportunidad muy importante para utilizar, toda vez que si bien se han tratado de realizar algunas acciones similares, se tiene por desgracia que en algunos países como México, Colombia, Brasil y Argentina, que los docentes se han cansado de participar en este tipo de escenarios, cuando sus propuestas no han sido escuchadas y bajo ninguna comunicación cla$\mathrm{ra}$, se les ha informado o retroalimentado por parte del Gobierno, porque sus propuestas no se han considerado al momento de construir o intervenir políticas públicas educativas.

Así las cosas, se propone que las mesas de trabajo en las que participan los docentes no sean ejercicios de discusión aislados de la agen- da pública, y más bien, como lo indica Barbosa (2004), se procura que la información consolidada desde estas iniciativas sea el elemento clave al momento de debatir sobre el futuro de la educación, tanto de los gobiernos latinoamericanos como del mundo entero, dejando claro que el docente no puede ser visto como un instrumento de la educación, y la docencia como profesión no puede ser instrumentalizada, sino que el docente debe ser visto como un agente de cambio que propone, articula, discute y posibilita que las políticas educativas se ejecuten en los términos y contextos en los que se han planeado.

\section{Procurar la participación sindical en temas de la agenda educativa}

En contextos como Latinoamérica no es desconocido que la fuerza sindical y las agremiaciones de trabajadores son fuerzas que convoca a muchas personas y movilizan gran cantidad de intereses, tanto es que, Pruzzo (2002) comparte que los gobiernos concretan con estas agrupaciones de trabajadores para debatir temas, como salario de trabajadores, condiciones laborales, beneficios en seguridad social, entre otros; es por esto que las agrupaciones educativas sindicales no son la excepción y desde la perspectiva educativa y gubernamental resulta clara la importancia e incidencia de considerar a estas fuerzas en las decisiones que se toman por parte de los gobiernos.

Luego de comprender la importancia de los sindicatos y agremiaciones del sector educativo para con la dinámica nacional de la mayoría de gobiernos latinoamericanos, Torres (1998) propone que la fuerza sindical no solo sea un movimiento a favor del mejoramiento de las condiciones laborales, sino que sea presentado como un movimiento a favor de toda la realidad educativa de los países, así las cosas, se propone que los gobiernos fomenten acuerdos de cooperación y gestionen alianzas para que la fuerza sindical no sea únicamente sinónimo de movimientos laborales, sino sea una fuente de propuestas no impositivas, desde las cuales 
los gobiernos puedan alimentar las intervenciones en la educación.

Esta propuesta requiere un manejo muy diplomático y a la vez cuidadoso, y como lo menciona Street (2003), estas acciones no solo buscan ampliar las brechas de las relaciones entre los gobiernos y los sindicatos, sino que puede llegar a ser una importante herramienta de gobierno con la cual se liman las asperezas que comúnmente se generan en los diálogos entre ambas partes $y$, adicional, puede ser un elemento clave que los docentes sean escuchados, tengan de por medio una fuerza colectiva que les incite a participar y ser protagonistas activos del cambio y las intervenciones a los sistemas educativos.

\section{Fortalecer el liderazgo educativo}

El liderazgo educativo es una premisa importante de la realidad de cualquier centro de educción, toda vez que, desde la capacidad del liderazgo que el director educativo tiene para con su comunidad educativa es que el cumplimiento de metas se alcance de forma conjunta, dejando claro que el destino de una institución de educación sin importar el nivel educativo al cual pertenezca, no es alcanzado únicamente por su director educativo, sino que se alcanza de forma conjunta con quienes él tiene a su lado.

Ahora bien, según Samper, Sanuy y Biscarri (1995) el fortalecer el liderazgo en una institución educativa favorece que el director educativo pueda movilizar a los docentes y la comunidad educativa en general, encaminados todos a la participación colectiva en la que se puedan contextualizar propuestas a favor de cambios en las políticas educativas, teniendo como premisa la realidad que se vive en las aulas y desde el quehacer docente y su labor.

Esta movilización desde la dirección de una institución de educación no es una tarea fácil, y tal como lo comparte Chiva-Bartoll y Gil-Gómez (2018), debe contar con persistencia y claridad para con la intención de la movilización que se procura, siendo clave que cada miembro de una comunidad educativa tenga claridad de su rol, el aporte para con la institución en la cual participa y cómo su labor contribuye, y que en conjunto cada docente sea participante constante y su labor sea considerada como una tarea indispensable para la sobrevivencia de un sistema educativo.

\section{Formación académica en la labor docente}

Cuando se hace referencia a formación académica se alude a calificación docente, desde crecimiento académico tanto formal como no formal, de manera que esta formación se configura en un aspecto más que importante en el quehacer docente, toda vez que, tal como lo da a conocer Asprelli (2012), desde una formación constante en el docente se pueden mejorar las estrategias, competencias y habilidades que día a día deben ser acordes al comportamiento mismo de la sociedad; dicho en otras palabras, la formación académica del docente debe dar cuenta de las situaciones que se viven en la sociedad y desarrollar procesos educativos del siglo XXI.

Para que esta formación académica sea constante y garantice que la labor del docente sea optima, se propone lo siguiente:

\section{Oferta educativa contextualizada}

El contexto en la educación, sin duda, es un elemento trascendental del éxito o no de un sistema educativo, llegando a ser conocido como el propósito mismo de la educación para con las necesidades y problemáticas que una población necesita en su día a día, de manera que así como lo menciona Marcano y Urbay (2008), la formación académica de los docentes debe dar cuenta de coherencia entre las necesidades de los estudiantes y comunidad en general y la oferta a seleccionar por el docente en su plan de formación académico. 


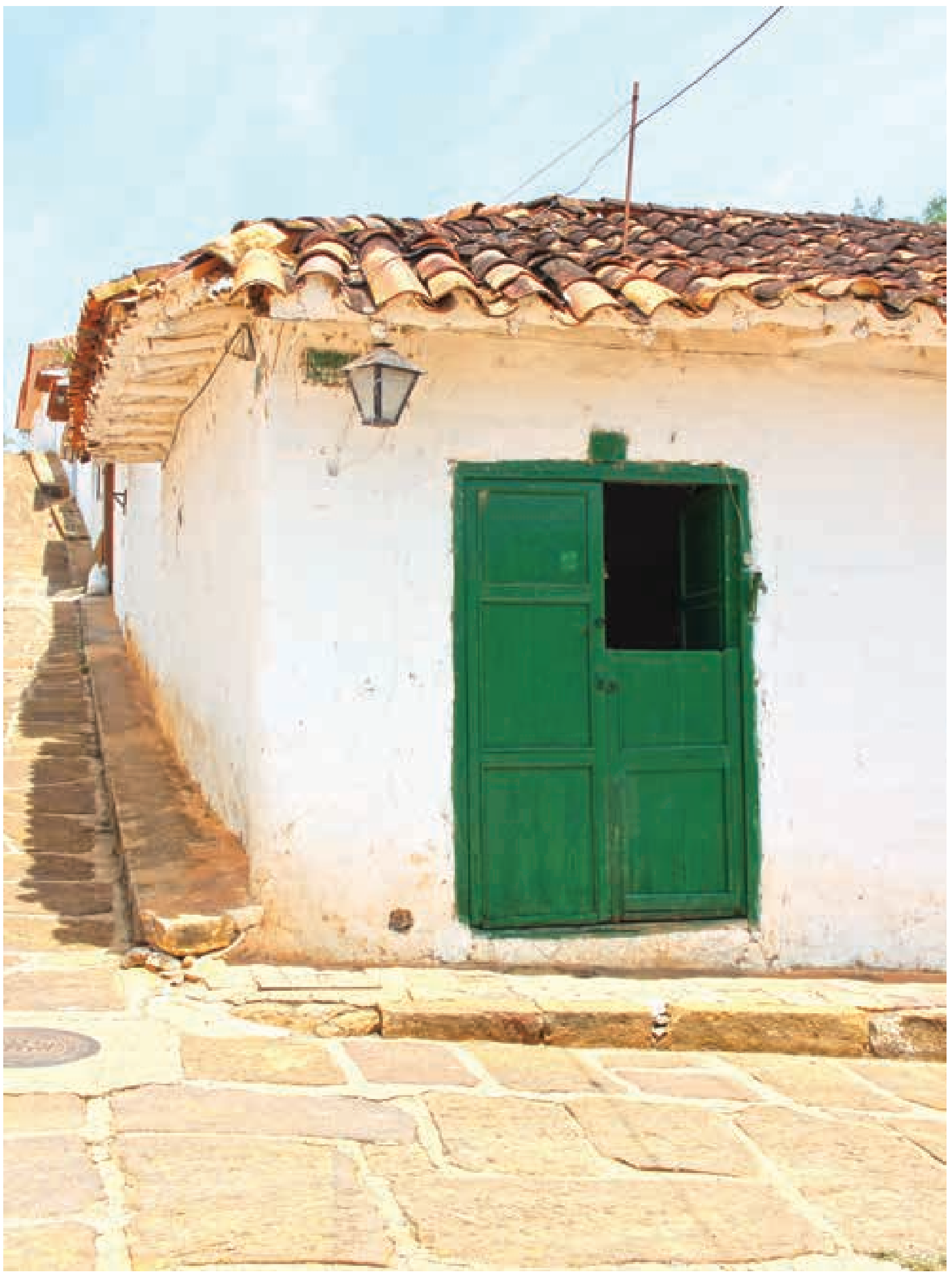


Este panorama para Cardelli y Duhalde (2002) pone en contexto dos realidades en las cuales el docente posiblemente se ve enfrentado, como son: a. existencia de oferta educativa acorde a lo que su labor y realidades ameritan, y b. intención de querer formarse según las necesidades de su población; ambas son el reflejo de lo que hoy en día se vive en Latinoamérica, dejando claro que en algunas poblaciones la oferta educativa se limita a la viabilidad económica que tienen las universidades para ofrecer programas de formación acordes a las necesidades de sus entornos, siendo una situación cada vez más cruda, en la cual algunos docentes deben seleccionar formación académica de posgrado que no es afín a sus contextos, optando por una limitada oferta educativa que obedece a intereses de sostenimiento económico y de número de estudiantes por parte de las universidades que ofrecen dichos programas.

Adicional, una problemática que se genera desde esta situación está presente en la carencia de vocación docente, teniendo como resultado que muchos docentes latinoamericanos han optado por esta labor como una ruta fácil para ocupar una plaza; y en la educación pública es muy cruda esta realidad, toda vez que, como lo da a conocer Flores, González y González (2008) es muy común encontrar docentes que reciben su salario del erario, pero no tienen el más mínimo sentido de pertenencia para con lo que significa esta labor, situación que conlleva cuestionar y replantear los mecanismos para ingresar a la carrera docente que han establecido la gran mayoría de países latinoamericanos.

Partiendo de esto, se propone que la formación académica de los docentes sin importar su vinculación ya sea en el sector público o privado, denote lo siguiente:

- La formación docente debe ser constante y en todo momento el docente debe estar actualizando sus conocimientos, de manera que no se puede pretender que la formación colectiva del conocimiento sea basada en las necesidades del siglo XX, con instituciones educativas que funcionan como en el siglo XIX, para estudiantes del siglo XXI.

- La oferta educativa de las universidades, si bien es en muchos casos es la única oferta con que se cuenta para la cualificación docente, también es claro encontrar que la educación a la medida se genera cuando los mismos sistemas educativos identifican necesidades por atender, y basado en esto, solicitan la apertura de programas educativos que dan respuesta a las necesidades del mismo sistema educativo.

Este panorama, invita a generar una importante intervención en la planeación educativa y en la caracterización de las poblaciones, de manera que, de forma conjunta las universidades, los centros educativos y los docentes, puedan trabajar para proponer soluciones a esas problemáticas no atendidas y se garantice la oferta educativa contextualizada desde la labor del docente para con sus comunidades.

\section{Plan de carrera determinado por la constante formación académica}

En contextos como el latinoamericano, es común encontrar situaciones en las que los docentes construyen para sí mismos un plan de carrera acelerado, en el cual por desgracia se demuestra una única intención de crecimiento, alineada con el mejoramiento salarial; y tal como lo menciona Rivas, Veleda y Mezzadra (2013), esta situación se presenta por lo permisivos que son muchos sistemas educativos, los cuales no han actualizado e intervenido con vehemencia los planes de crecimiento en el plan de carrera docente, situación que conlleva ratificar que en muchos casos el funcionamiento administrativo de los centros educativos, y los mismos sistemas educativos, parecen estar funcionando con políticas y lineamientos de dos siglos atrás. 
Para esto, cabe identificar, por ejemplo, lo que indica Cardelli y Duhalde (2002), quienes mencionan que la formación posgradual a nivel de doctorado y maestría, en muchos casos no es coherente con producción investigativa por parte de quienes se están formando y quien se ha formado, siendo un claro ejemplo de formación de posgrado que se ha cumplido como requisito para crecer en el llamado escalafón docente, pero no da resultados por la razón misma del nivel educativo alcanzado, la cual esta alienada con producción investigativa.

Así las cosas, se propone que el plan de carrera docente, sin importar su nivel de formación, esté alienado coherentemente con su formación académica, siendo la formación un requisito para hacer parte y luego de hacer parte, continuar siéndolo; de forma que, quien ingrese a la carrera docente no puede únicamente quedarse con un programa posgradual en su hoja de vida, adicional debe demostrar continuamente producción académica, generada propiamente de su labor como el fruto de su aporte a la sociedad y como el resultado mismo de su vocación.

El plan de carrera docente, para Díaz, Blázquez, y Casse (2007) debe ser presentado de forma obligatoria al docente como una oferta educativa que aporte en su labor, favoreciendo que se rompan las zonas de confort en las cuales muchos docentes han caído con el paso del tiempo, adicional, se debe dejar claro que no se procura la acumulación de titulaciones, sino la actualización de conocimientos, perfeccionamiento de destrezas y adquisición de nuevas habilidades, las cuales se van construyendo y midiendo a medida que el docente crece laboralmente y optimiza su labor.

\section{Nuevas competencias en el quehacer docente}

La labor del docente del siglo XIX con total seguridad ha experimentado cambios para con el quehacer del docente del siglo XX y del siglo
XXI, lo cual conlleva cuestionar las habilidades con que muchos docentes están realizando su labor, y precisamente desde la perspectiva latinoamericana como lo indica Oliveira, Gonçalves y Melo (2004), esta situación presenta grandes retos, retos que sin lugar a dudas de no ser intervenidos generaran día a día una brecha que segrega a las comunidades, aísla las poblaciones $y$, sin lugar a equivocación, impactará negativamente los sistemas educativos.

Para comprender cuáles son estas nuevas competencias, basta con observar el mundo actual y la globalización misma de las sociedades, cuya intervención es clara de evidenciar en esta época contemporánea, teniendo como reto para el docente el ampliar sus conocimientos en temáticas como:

\section{- Tecnologías de información y comunicación}

Las llamadas TIC, no solo están presentes en las comunicaciones y los negocios, también han llegado para quedarse en los procesos educativos y con seguridad el futuro de los procesos educativos actuales, dependerán en el mediano y largo plazo en gran medida de la forma como se interactúa con estas tecnologías, ante lo cual Rueda (2013) menciona que esto presenta un sin número de posibilidades por mejorar y optimizar las actuales actuaciones de los docentes, salvo que en contextos, como el latinoamericano en pleno siglo XXI, el concepto de analfabetismo digital aún está presente en gran parte de sus docentes, docentes que ejercen su labor en todos los niveles educativos, desde preescolar y educación inicial, pasado por la educación primaria, media, bachillerato y todo el conjunto de niveles que agrupan la educación superior.

El reto que los sistemas educativos asumen es grande, más cuando es claro que no se puede cambiar a toda la nómina docente que no cuente con estos conocimientos en TIC, salvo que si se pueden generar programas de actualización docente que posibiliten la cualificación 
docente en estos temas, y se procure la actualización docente que sea permanente desde en las dinámicas que hoy se viven en la sociedad, que van desde la tecnología, la información, la ciber-seguridad, sostenibilidad, hasta conceptos totalmente nuevos como nanomedicina, ingeniería biomédica, entre otros.

Si bien las TIC presentan a la vez el concepto de comunicación, este concepto por parte del docente debe ser interiorizado, no solo como el proceso comunicativo, sino como el conjunto de acciones en las que la humanidad se está comunicando e intercambiando información, por esto es muy necesario que el conocimiento de nuevos idiomas y culturas, sea adquirido por parte de los docentes, quienes en resumen están posibilitando que la educación tenga utilidad para con las problemáticas sociales y continúe siendo la labor que materializa todas las profesiones.

\section{- El docente frente a los procesos investigativos}

El docente latinoamericano por desgracia como lo indica Fiorito (2009), es uno de los docentes con menor participación investigativa en el escenario mundial desde la perspectiva académica, lo cual no puede ser desconocido por los sistemas educativos y sí genera un panorama enorme por: a. enseñar al docente a investigar, para que posterior, desde su labor, tenga las habilidades para fomentar de igual forma la investigación en sus estudiantes, b. favorecer que desde su labor pueda investigar, c. encontrar en la investigación la respuesta a las necesidades que se le presenta en su entorno, y d. hacer que las investigaciones tenga utilidad al ser públicas y comunicadas a la comunidad educativa mundial.

La construcción de una cultura investigativa desde el docente requiere bastante tiempo, más cuando posiblemente el docente no ha sido partícipe de una comunidad investigativa, lo cual hace que precisamente la docencia tenga un valor agregado destacado desde su quehacer, en el cual existe la obligatoriedad por reinventarse y evitar la monotonía; por lo cual Palladino (1995) menciona que la investigación en el aula debe ser un pilar fundamental para el docente, quien integra sus conocimientos en pro de problemáticas actuales y contribuye en posibles soluciones a estas realidades, dejando claro que, antes que nada, tiene la capacidad por aprender y desprender o mejor dicho, actualizar sus conocimientos para con las nuevas épocas.

\section{- La gestión de habilidades blandas}

En la contemporaneidad por la cual está atravesando la humanidad, no resulta desconocido que algunos ciudadanos compartan anécdotas en las cuales se afirme que la humanidad va a ser remplazada por la tecnología y que en un futuro no muy lejano, la tecnología dominará al hombre; siendo pertinente el aporte de GoigMartínez (2014), quien indica que frente a esto el ser humano en los últimos años ha encontrado que su humanidad misma es la que le garantizará la supervivencia en los años venideros, a partir de esto, la educación de las nuevas generaciones y de las generaciones actuales necesita obligatoriamente el comprender que son las habilidades blandas y como estas permitirán que el hombre sobreviva a las adversidades.

En el contexto latinoamericano, las habilidades blandas posiblemente encuentran un entorno más dócil para florecer respecto del mundo entero, toda vez que la cultura y los aspectos únicos de la comunidad latina, desde lo global, están basados gran parte en algunas de estas habilidades, las cuales siempre han estado en el día a día, salvo que aun así, resulta necesario adquirir otras habilidades que no se han cultivado de igual forma, frente a esto es claro, en primera instancia, que el docente adquiera estas habilidades para que las pueda construir colectivamente con sus estudiantes y las futuras generaciones puedan contar con estas en sus relaciones humanas. Frente a las 
habilidades blandas que posiblemente mayor protagonismo deban tener, están: a. comunicación asertiva, b. trabajo en equipo, c. adaptación al cambio, d. toma de decisiones, e. capacidad de escucha, y f. manejo de emociones.

\section{Conclusiones}

El panorama mundial está presentando múltiples retos a la humanidad, es por esto que la docencia no es la excepción y ante esto requiere que sus docentes estén constantemente evaluando las estrategias pedagógicas usadas en el aula, considerando los cambios del entorno para enriquecer sus actividades curriculares y académicas; de igual forma le representa un reto a los gobiernos que deben procurar la constante evaluación y optimización de las políticas públicas educativas, de manera que, de forma conjunta la humanidad no pueda desconocer las dinámicas del mundo.
Desde la perspectiva del docente, es más que necesaria su participación en la construcción de políticas educativas, generación de propuestas para intervenir los sistemas educativos, construcción de entornos de debate abierto para escuchar las partes y antes que nada, tener claro que la labor del docente es articuladora en el interior de cada centro de educación y para con los sistemas educativos, los docentes cobran suma importancia al ser quienes posibilitan la ejecución y materialización de las metas y objetivos planteados por los gobiernos en términos educativos.

Es relevante mencionar que, el docente debe estar constantemente actualizándose, y esta actualización puede ser con educación formal y no formal, lo cual significa que puede ser desde formación de posgrado hasta educación continuada, cuya finalidad tenga como resultado aportes en la mejora del desempeño

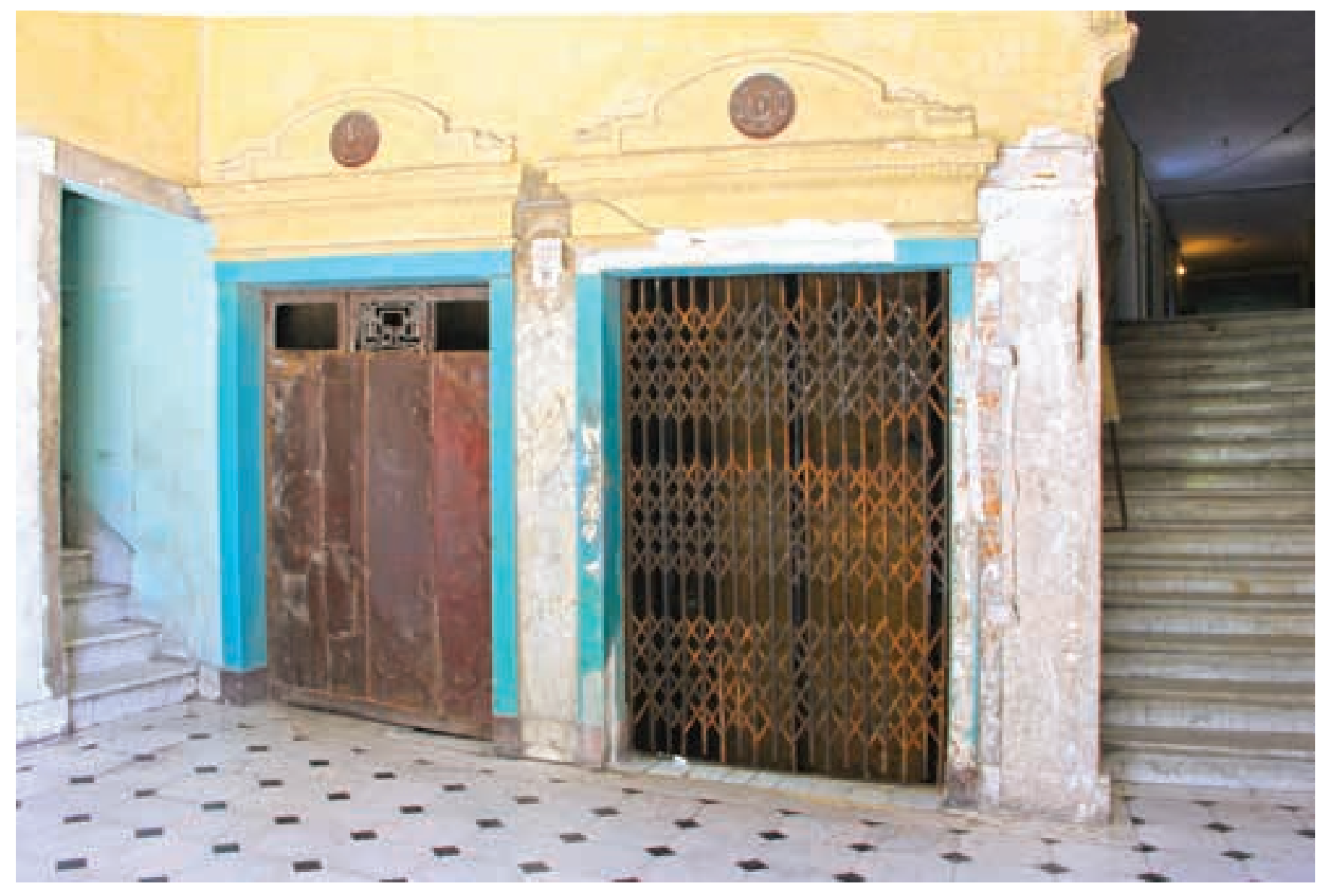


de las instituciones educativas y de los sistemas educativos, reiterando que el escenario latinoamericano no puede continuar presentando situaciones en las que los docentes quizás solo acumulan titulaciones para mejorar sus condiciones laborales, sino que la formación académica que se obtiene, a la vez presente resultados medibles y de impacto en las comunidades.

De igual forma, se concluye que los docentes deben estar fortaleciendo nuevas competencias contemporáneas, las cuales posibilitan que la labor del docente y la profesión docente tenga la pertinencia que la sociedad actual requiere, por lo tanto, no se puede aceptar que la profesión docente sea instrumentalizada, ya que el ser humano no puede instrumentalizar su humanidad misma, de allí que el docente así como lo ha sido, lo es y lo seguirá siendo, es el encargado de aportar directamente en la mejora del desempeño de las nuevas generaciones, en la construcción de una sociedad más justa y equitativa, representado un desafío para quien asume ser docente, toda vez que la profesión docente integra a toda la sociedad y el futuro de las civilizaciones sobre esta recae.

\section{Referencias}

Asprelli, M. (2012). La didáctica en la formación docente. Santa Fe: Homo Sapiens Ediciones. Recuperado el 30/10/2019, de https://ebookcentral.proquest.com

Barbosa, M. (2004). En las redes de la profesión: resignificando el trabajo docente. Revista Mexicana de Investigación Educativa, 159-181. Recuperado el 30/10/2019 de https://ebookcentral.proquest.com

Cardelli, J., y Duhalde, M. (2002). Didáctica de la investigación, Una experiencia de formación docente. Buenos Aires: Miño y Dávila. Recuperado el 30/10/2019, de https:// ebookcentral.proquest.com

Chiva-Bartoll, Ó., y Gil-Gómez, J. (2018). Aprendizaje-servicio universitario: modelos de intervención e investigación en la formación inicial docente. Barcelona: Ediciones Octaedro, S.L. Recuperado el 30/10/2019, de https://ebookcentral.proquest.com

Díaz, F., Blázquez, P., y Casse, J. (2007). Modelo para autoevaluar la práctica docente. Madrid, España: Wolters Kluwer. Recuperado el 30/10/2019, de https://ebookcentral.proquest.com

El Plan Maestro. (2015). Propuestas participativas para una nueva profesión docente. Santiago de Chile: RIL Editores. Recuperado el 30/10/2019, de https://ebookcentral.proquest.com
Fiorito, M. (2009). Enseñar, proyectar, investigar: experiencias y reflexiones de la carrera de formación docente. Buenos Aires: Editorial Nobuko. Recuperado el 15/11/2019, de https://ebookcentral.proquest.com

Flores, F., González, F., y González, A. (2008). Las competencias que los profesores de educación básica movilizan en su desempeño profesional docente. Madrid: Universidad Complutense de Madrid. Recuperado el 15/11/2019, de https://ebookcentral.proquest.com

Gisbert, M., Esteve-González, V., y Lázaro, J. (2019). ¿Cómo abordar la educación del futuro?: conceptualización, desarrollo y evaluación desde la competencia digital docente. Barcelona: Ediciones Octaedro, S.L. Recuperado el 30/10/2019, de https://ebookcentral.proquest.com

Goig-Martínez, R. (2014). Formación del profesorado en la sociedad digital: investigación e innovación y recursos didácticos. Madrid: UNED - Universidad Nacional de Educación a Distancia. Recuperado el 30/10/2019, de https:// ebookcentral.proquest.com

Lozano, I., y Mercado, E. (2011). El ojo del huracán: la formación y la práctica del docente de secundaria: miradas divergentes. México, D.F.: Ediciones Díaz de Santos. Recuperado el 15/11/2019, de https://ebookcentral.proquest.com

Marcano, L., y Urbay, M. (2008). Modelo de evaluación del desempeño profesional docente como vía para el desarrollo profesional. Ciudad de La Habana: Editorial Universitaria. Recuperado el 30/10/2019, de https://ebookcentral. proquest.com

Marcelo, C. (2010). El profesorado principiante: inserción a la docencia. Barcelona: Ediciones Octaedro, S.L. Recuperado el 30/10/2019, de en: https://ebookcentral.proquest. com

Matute, E., y Romo, R. (2000). Diversas perspectivas sobre la formación docente. Guadalajara: Editorial de la Universidad de Guadalajara. Recuperado el 30/10/2019, de https://ebookcentral.proquest.com

Medina, A., Pérez, L., y Campos, B. (2014). Elaboración de planes y programas de formación del profesorado en didácticas especiales. Madrid: UNED - Universidad Nacional de Educación a Distancia. Recuperado el 30/11/2019, de https://ebookcentral.proquest.com

Medina-Alvarado, I. (2019). Economía de la educación y el comportamiento del profesorado en el mercado laboral latinoamericano. Revista Caribeña de Ciencias Sociales, 1-13. Recuperado el 30/11/2019, de https://www. eumed.net/rev/caribe/2019/11/profesorado-mercadolaboral.html

Oliveira, D., Gonçalves, G., y Melo, S. (2004). Cambios en la organización del trabajo docente: consecuencias para los profesores. Revista Mexicana de Investigación Educativa, 183-197. Recuperado el 30/12/2019, de https:// ebookcentral.proquest.com

Palladino, E. (1995). Investigación educativa y capacitación docente: investigación sobre perfil docente. Buenos Aires: Espacio Editorial. Recuperado el 30/10/2019, de https:// ebookcentral.proquest.com

Pruzzo, V. (2002). Transformación de la formación docente: desde las tradicionales prácticas a las nuevas ayudantías. Buenos Aires: Espacio Editorial. Recuperado el 15/12/2019, de https://ebookcentral.proquest.com 
Rivas, A., Veleda, C., y Mezzadra, F. (2013). Caminos para la educación: bases, esencias e ideas de política educativa. Buenos Aires: Ediciones Granica. Recuperado el 30/11/2019, de https://ebookcentral.proquest.com

Rueda, M. (2013). La evaluación educativa: análisis de sus prácticas. México D.F.: Ediciones Díaz de Santos. Recuperado el 30/12/2019, de https://ebookcentral.proquest. com

Samper, L., Sanuy, J., y Biscarri, J. (1995). Movilidad vertical en la carrera docente. Lérida: Edicions de la Universitat de Lleida. Recuperado el 30/11/2019, de https://ebookcentral.proquest.com
Street, S. (2003). Trabajo docente y subjetividad magisterial: viejos debates en nuevos contextos. Revista Mexicana de Investigación Educativa, 599-602. Recuperado el 15/11/2019, de https://ebookcentral.proquest.com

Torres, R. (1998). Nuevo papel docente. ¿Qué modelo de formación y para qué modelo educativo? Revista Perfiles Educativos, 1-20. Recuperado el 15/11/2019, de https:// ebookcentral.proquest.com

Vaillant, D., y Marcelo, C. (2015). El ABC y D de la formación docente. Madrid: Narcea Ediciones. Recuperado el 30/10/2019, de https://ebookcentral.proquest.com 